\title{
Use of 3D Images to Evaluate Formation Damage Induced by Montmorillonite Fines in Porous Media Systems
}

\author{
Jamal Hannun \\ jh1300283@qu.edu.qa \\ Department of Civil and Architectural Engineering, Qatar University, Doha, Qatar

\section{Riyadh Al-Raoush} \\ riyadh@qu.edu.qa \\ Department of Civil and Architectural Engineering, Qatar University, Doha, Qatar \\ Zaher Jarrar \\ zjarrar@vols.utk.edu \\ Civil and Environmental Engineering, University of Tennessee, Knoxville, TN 37996, USA
}

\author{
Khalid Alshibli \\ alshibli@utk.edu \\ Civil and Environmental Engineering, University of Tennessee, Knoxville, TN 37996, USA \\ Jongwon Jung \\ jjung@chungbuk.ac.kr \\ School of Civil Engineering, Chungbuk National University, Cheongju, Chungbuk, South Korea
}

\begin{abstract}
Formation damage costs oil and gas industry $\$ 140$ billion/year in lost productivity, this is a key challenge to Qatar, the world's largest LNG exporter. During production from a well, multiphase flow foster drag forces to mobilize fine particles from within the subsurface. Fines' migration can alter the gas flow, clogging pores and disconnecting gas pathways. Understanding fines influence is a complex challenge due to the reservoirs' porous media heterogeneity. Microtomography of sand sediments provides a standardized approach to study the fines impact. X-Ray microtomography of two repacked sand cylinders was carried at Argonne National Lab synchrotron. Rounded silica sand was mixed with hydrophilic swelling montmorillonite clay. High and low fines concentrations were $\operatorname{mix} \neg \neg$ ed with the sand then deposited into five layers. Initially, samples were fully saturated, then gas was injected, the sediments were scanned before and after injection. At first, fines were suspended in the brine, but after injection were retained on the gasbrine interface, and their concentration in the brine increased. Gas injection divided pores and throats, reducing their average size. Contrarily, main gas pathways increased in size but were disconnected in the sediment with high fines concentration. Fines caused increased capillary pressure and lowered the sediment permeability.
\end{abstract}

Keywords: Geopolymer; Fluid to binder ratio; Activator solution; $\mathrm{Na} 2 \mathrm{SiO} 3 / \mathrm{NaOH}$ ratio

\section{INTRODUCTION}

Multiphase flow of brine and gas in unconsolidated sandy porous media, have an influence on broad set of natural and industrial activities at the subsurface. Methane hydrate, fracking, rainwater and contaminants transported in the vadose zone, are all applications that are dominated by multiphase flow mechanisms in the subsurface. 
Dominantly, capillary forces control the horizontal flow of fluids in sandy sediments. The physics of fluids are governed by pore sized pathways, the geometry of these pathways can affect the flow mechanisms. During gas production from deep rock formations, spontaneous imbibition drives the flow, this flow is governed by the pore volumes in the rock formation and is not caused by pressure gradient within the reservoir. Over time, formation damage occurs within rock pores changing their size, this can seize the spontaneous imbibition, causing the need for injection wells to continue production. Understanding the different mechanisms of formation damage is fundamental in planning any activity that involves the multiphase flow of fluids in the subsurface. It is reported that formation damage costs the oil and gas industry $\$ 140$ billion/year in lost productivity (Byrne, 2012). Lost productivity is a key challenge to Qatar, the world's largest LNG exporter. During production from a well, multiphase flow foster drag forces to mobilize fine particles from within the subsurface. Fines' migration can alter the gas flow, clogging pores and disconnecting gas pathways, changing pathways size and geometry. Understanding fines influence is a complex challenge due to the reservoirs' porous media heterogeneity. The study of homogenous sand sediments provides a standardized approach to benchmark and understand the fines impact. Understanding the quantitative and qualitative effects of fines in the subsurface is fundamental to advance current gas recovery systems and evolve sustainable production technics. Also, it is crucial to conduct such studies in three-dimensional sediments, microtomography is a powerful method that enables accurate reconstruction of real sediment morphology.

\section{METHODOLOGY}

$\mathrm{X}$-ray microtomography of two repacked sand cylinders was carried at Argonne National Lab (ANL) synchrotron. Rounded silica sand was mixed with hydrophilic swelling montmorillonite clay. High and low fines concentrations were mixed with the sand then deposited into five layers, into two different acrylic cylinders. Initially, samples were fully saturated, then $\mathrm{CO}_{2}$ gas was injected, the sediments were scanned before and after injection. The scan was done using high brilliance X-rays generated from the synchrotron facility at beamline 13D, Advanced Photon Source (APS), (ANL), Illinois, the USA. A monochromatic beam was pointed towards the sample as it rotated, the scan time took 15 minutes for each sample. Gas was injected, then imaged in the quasi-static state. A scan resolution of 3.9 micron per voxel was chosen for this study, this resolution can resolve the movements of clusters of fines, whether suspended in brine or attaching to the sand interface, changing the pore morphology.

Using the resulting 3D X-ray grayscale images, sand, brine, gas, and fines were binarized to observe the qualitative changes due to the transport of fines. The segmented images were then used to observe changes in the gas flow pattern. As fines concentration increases, capillary flow mechanisms can be altered due to changes in pore morphology (Al-Raoush et al., 2019). These changes can be visually examined by visualizing the 3D gas volume.

Then pore networks were generated to study the quantitative changes in the porous media morphology. A pore network algorithm that uses the maximum spheres approach was used for this analysis (Fouard et al., 2006). The algorithm starts by constructing a skeleton of the porous media that represents the central line of the flow paths, then the 
intersection points are grown to spheres in $3 \mathrm{D}$, the inscribed sphere radius increases up until it touches a sand grain. Figure 1 shows a vertical section of the media, (a) is void space in blue, (b) is a distant map used for pore network generation, colored blue toward red as the distance between central line and grains increases (indicating bigger pore radius). Changes in the average pore size are then examined to study the impact of swelling hydrophilic montmorillonite clay on the porous media, due to multiphase flow of the injected gas.

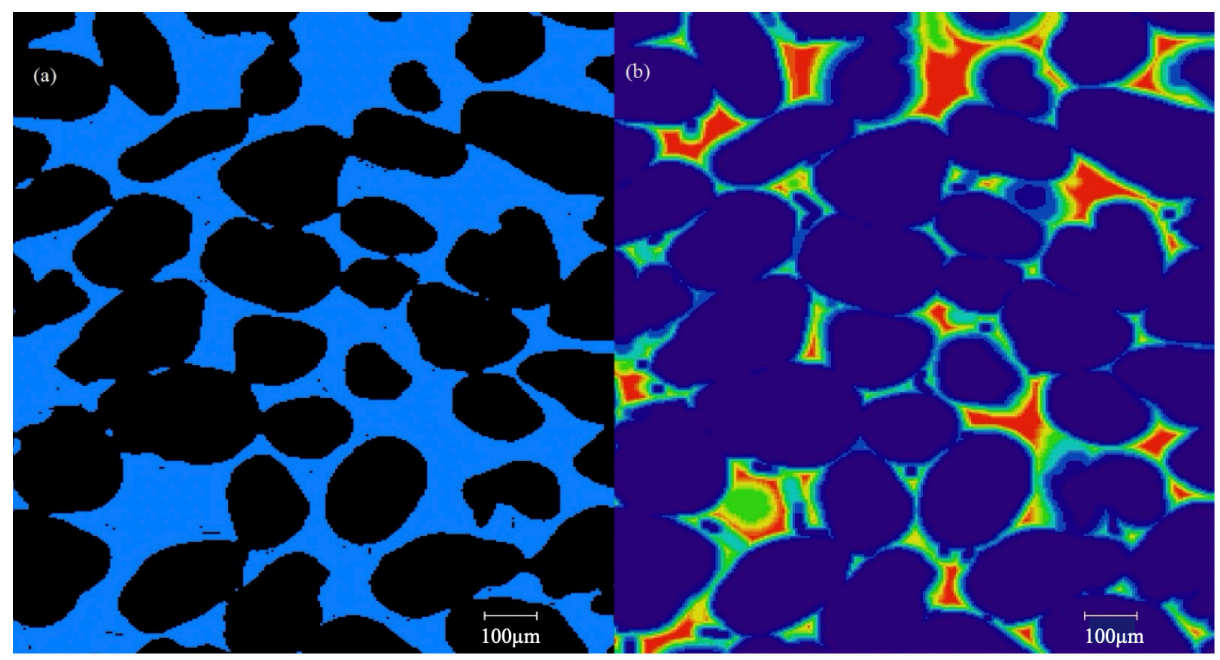

Figure 1: Vertical section of the sediment pack, (a) void space, (b) distant map

\section{RESULTS}

The results for the two systems, low and high fines concentrations showed a change in gas flow patterns and average pore sizes. The gas flow pattern in the $2 \%$ montmorillonite (concentration by weight of sand in the system), was a capillary flow in which a connected gas ganglion was observed, this was validated by visualizing the gas volume. In the other sample with 5\% montmorillonite, the gas body was observed to disconnect after $\mathrm{CO}_{2}$ injection, this can be related to the mobilization of fines clogging the pore throats. The generated pore networks provide a measure for the size change of all the pores in the system. Comparing the change of the average pore size after $\mathrm{CO}_{2}$ injection, can provide a justification for the gas pathways disconnection in the 5\% sample. Table 1 shows how fines reduced the pores and throats sizes causing detachment in the gas ganglion.

These results using maximal ball based pore networks are similar in behavior to other results obtained using grain-based algorithms (Hannun et al., 2019). Figure 2 illustrates the generated maximal ball pore network, (a) is the void space, (b) pore network and (c) is an overlap showing how the network fits the void space. The fines transported in the subsurface can alter the pores morphology, this change is the main cause in altering gas flow patterns.

Fines were suspended in the brine, but after injection were retained on the gas-brine interface, and their concentration in the brine increased. Fines caused increased capillary pressure and lowered the sediment permeability. This is primarily due to the decrease of 
pore sizes after the migration of the swelling hydrophilic clay.

Table 1: Change in average pore radius at different fines concentrations due to $\mathrm{CO}_{2}$ injection

\begin{tabular}{|c|c|c|c|}
\hline Fines Type & Fines\% & Injected $\mathbf{C O}_{2}(\mathbf{m l})$ [Change in Average Pore Radius] \\
\hline Montmorillonite & 2 & $0[100 \%]$ & $1.5[99.98 \%]$ \\
\hline Montmorillonite & 5 & $0[100 \%]$ & $1.5[97.80 \%]$ \\
\hline \multicolumn{2}{|c|}{ Gauge CO Pressure psi(kPa) } & $4(27.6)$ & $6(41.4)$ \\
\hline
\end{tabular}
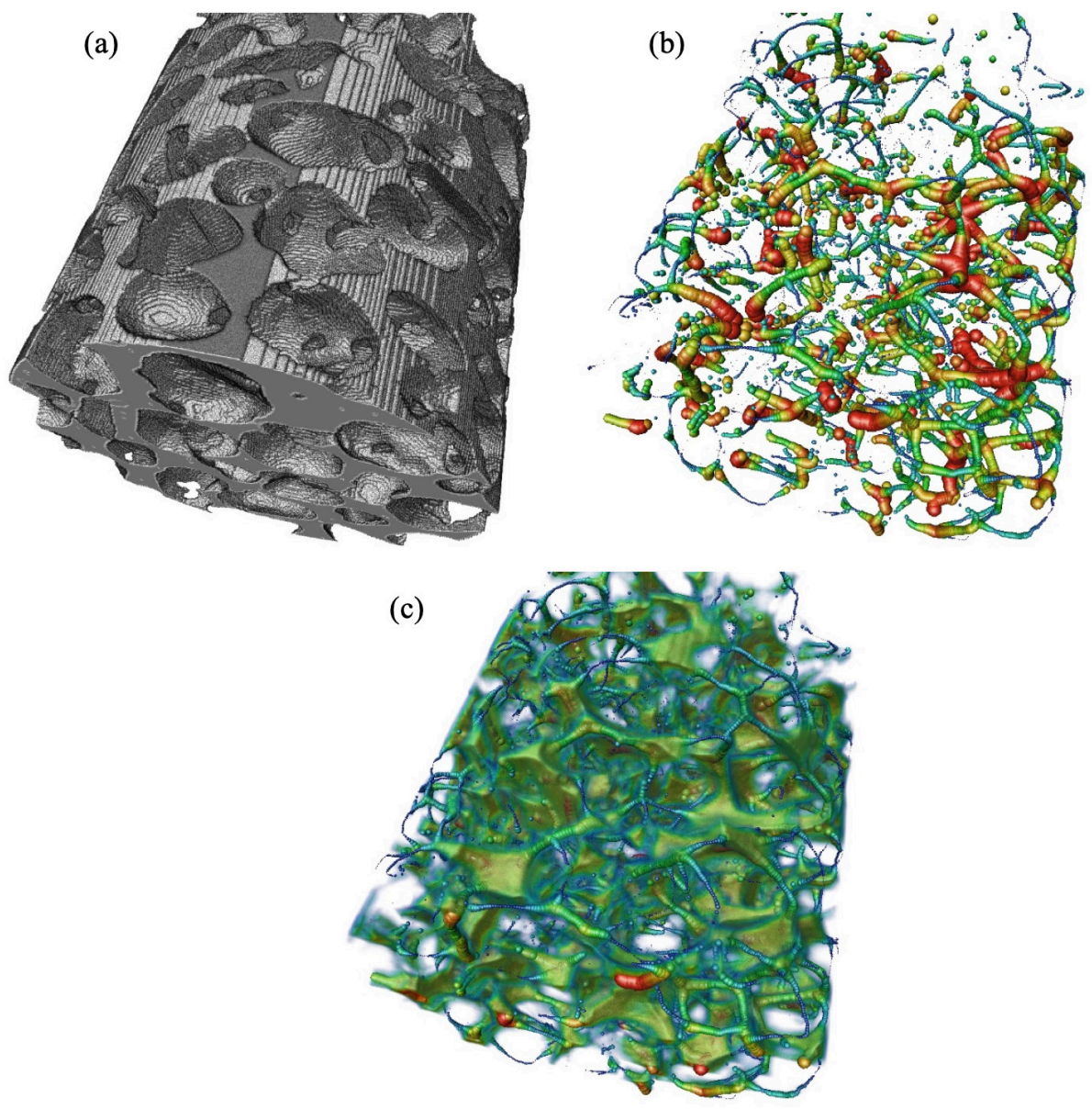

Figure 2: Maximal ball pore network, (a) the void space, (b) pore network, (c) overlap displaying; the network fitting the void space, blue to red are small to large pores respectively

\section{CONCLUSION}

Synchrotron microtomography of two sand samples was used to study the formation damage caused by fines' migration. Unconsolidated sand was mixed with low and high concentrations of montmorillonite clay, the samples were fully saturated then gas 
was injected to cause fines' mobilization. Using 3D X-ray image, pore networks were generated to provide a measure of the change in pore morphology due to fines' migration. Gas injection divided the pores and throats, reducing their average size. For the system with high fines concertation, the average pore radius was reduced by $2.2 \%$, compared to $0.02 \%$ in the system with lower fines concentration. This reduction of the throat radius resulted in increased capillary pressure and reduced permeability.

\section{ACKNOWLEDGMENTS}

This research was made possible by the National Priorities Research Program (NPRP) grant \#NPRP8-594-2-244 from Qatar national research fund (a member of Qatar Foundation). The findings achieved herein are solely the responsibility of the authors. The SMT images were collected using the X-ray Operations and Research Beamline Station 13-BMD at Argonne Photon Source (APS), Argonne National Laboratory. The authors thank Dr. Mark Rivers of APS for help in performing the SMT scans. They also acknowledge the support of GeoSoilEnviroCARS (Sector 13), which is supported by the National Science Foundation, USA, Earth Sciences (EAR-1128799), and the US Department of Energy (DOE) and Geosciences (DE-FG02-94ER14466). Use of the Advanced Photon Source, an Office of Science User Facility operated for the DOE Office of Science by Argonne National Laboratory, was supported by DOE, USA under contract no. DE-AC02-06CH11357.

\section{REFERENCES}

Al-Raoush, R., Hannun, J., Jarrar Z., Alshibli, K. \& Jung, J. (2019). Impact of fines type on gas flow using 3D micro-computed tomograph. SPE Kuwait Oil \& Gas Show and Conference. Society of Petroleum Engineers, Mishref, Kuwait. https://doi.org/10.2118/198106-MS.

Byrne, M. (2012). Formation damage - any time, any place, anywhere. http://www.afes.org.uk/ uploads/files/Formation\%20Damage $\% 20 \%$ E2\%80\%93\%20Any\%20Time.pdf.

Fouard, C., Malandain, G., Prohaska, S. \& Westerhoff, M. (2006). Blockwise processing applied to brain microvascular network study. IEEE Transactions on Medical Imaging, 25 (10), 131928. https://doi.org/10.1109/TMI.2006.880670.

Hannun, J., Al-Raoush, R., Jarrar Z., Alshibli, K. \& Jung, J. (2019). Pore networks to characterize formation damage due to fines at varied confinement and sand shape. Third EAGE WIPIC Workshop: Reservoir Management in Carbonates, Nov. 2019, Volume 2019, European Association of Geoscientists \& Engineers, Doha, Qatar. p.1-4, https://doi.org/10.3997/22144609.201903116. 